\title{
Gonadal Dysgenesis
}

National Cancer Institute

\section{Source}

National Cancer Institute. Gonadal Dysgenesis. NCI Thesaurus. Code C61420.

A congenital disorder characterized by the presence of extremely hypoplastic gonads preventing the development of secondary sex characteristics. 